\title{
CREACIÓN DE UN PROTOTIPO PARA LA EDICIÓN DIGITAL DEL CANCIONERO DE GÓMEZ MANRIQUE BASADO EN LA APLICACIÓN DE TEI A LA TRANSCRIPCIÓN Y CORRESPONDENCIA CON EL FACSÍMIL ${ }^{1}$
}

\author{
CREATION OF A PROTOTYPE FOR THE DIGITAL EDITION \\ OF GÓMEZ MANRIQUE'S CANCIONEROBASED ON THE APPLICATION \\ OF TEI TO THE TRANSCRIPTION AND CORRESPONDENCE WITH THE FACSIMILE
}

\author{
Andrea IANTORNO \\ Università degli studi della Tuscia, Viterbo (Italia) \\ andrea.iantorno@gmail.com
}

Resumen: En este trabajo se presenta una edición digital de un fragmento del Cancionero de Gómez Manrique, creada utilizando el estándar TEl (Text Encoding Initiative) para etiquetar semánticamente el texto, y los lenguajes XSL (eXtensible Style Sheets Language), CSS (Cascading Style Sheets), HTML5 (Hyper Text Markup Language) y jQuery para desarrollar la parte de presentación al usuario, donde se ha incluído también un sistema de transcripción y correspondencia con el facsímil.

Abstract: This paper presents a digital edition of a fragment of Gómez Manrique's Cancionero, created using TEl (Text Encoding Initiative), to markup the text semantically, and XSL (eXtensible Style Sheets Language), CSS (Cascading Style Sheets), HTML5 (Hyper Text Markup Language) and jQuery to develop the part of the user presentation, which also includes a transcription system and its correspondence with the facsimile.

Palabras clave: Humanidades Digitales. XML. TEI. XSL. CSS. HTML.

Key Words: Digital Humanities. XML. TEI. XSL. CSS. HTML.

1 Este artículo se ha llevado a cabo en el marco del proyecto de investigación FFI2014-57961-R, “Laboratorio de Innovación en Humanidades Digitales: edición digital, datos enlazados, y entorno virtual de investigación para el trabajo en humanidades" dirigido por la Dra. Elena González-Blanco. 


\section{INTRODUCCIÓN}

La edición digital de una parte del Cancionero de Gómez Manrique² (1412-1490) es el resultado de un proyecto de colaboración entre el Laboratorio de Innovación en Humanidades Digitales de la UNED (LINHD: http://linhd.uned.es)3, y la Real Biblioteca4.

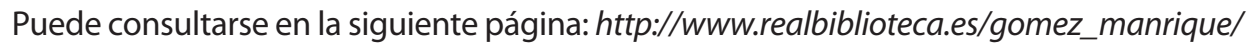
cancionero.html. El resultado es un prototipo que comprende la selección de un fragmento significativo de dicho Cancionero: los folios 11-44, para cuya transcripción nos hemos basado en la edición de $2013^{5}$. El código del proyecto se encuentra disponible en https://github.com/Andrealantorno/Cancionero-digital-de-G.-Manrique.

El desarrollo tecnológico llevado a cabo tiene como punto de inspiración y modelo el realizado en el proyecto Digital Vercelli Book (http://vbd.humnet.unipi.it/beta), pionero en su área, y comenzado en 2003 por iniciativa de Roberto Rosselli Del Turco, investigador de Filología Germánica en la Universidad de Turín.

El proyecto que aquí se presenta se desarrolla en varias fases que suponen la aplicación de capas tecnológicas que van actuando sobre las anteriores. Así, se comienza con la transcripción y etiquetando semánticamente el texto utilizando el estándar TEIXML (Text Encoding Initiative), creado para la representación de textos en forma digital, para posteriormente transformar y visualizar el etiquetado XML en una página HTML (Hyper Text Markup Language) a través de una hoja de estilo XSL (eXtensible Style Sheets Language).

TEl es un estándar "de facto", que se expresa a través de XML (eXtensible Markup Language), un lenguaje de marcado descriptivo que actualmente es el más utilizado para la marcación de textos humanísticos y que se convirtió en una Recomendación del W3C el 10 de febrero de 1998. Dentro del conjunto de etiquetas que TEI ofrece, en este proyecto se ha utilizado fundamentalmente el módulo 6, "Verse" (http://www.tei-c.org/release/doc/tei-p5-doc/es/html/VE.html). que "is intended for use when encoding texts which are entirely or predominantly in verse", (http://www.tei-c.org/release/doc/tei-p5-doc/es/html/VE.html). El etiquetado se ha llevado a cabo gracias al software oXygen XML Editor (http://www. oxygenxml.com/) que permite la edición en múltiples lenguajes basados en $\mathrm{XML}$, su transformación y desarrollo.

2 La signatura del manuscrito conservado en la Real Biblioteca de Madrid es II/1250.

3 Representado por su Directora y profesora del departamento de Literatura Española y Teoría de la Literatura en la Facultad de Filología de la UNED, Elena González-Blanco García.

4 Representada por José Luis Rodríguez Gómez, Técnico Superior de Automatización de dicha institución.

5 Manrique, Gómez, Cancionero: Manuscrito 1250 de la Biblioteca del Palacio Real. Edición de José I. Suárez, New York: National Hispanic Foundation for the Humanities, 2013. 
En primer lugar, es necesario tener en cuenta las reglas fundamentales de XML:

1. Con XML pueden inventarse etiquetas propias (por eso se llama eXtensible), siempre poniéndolas entre paréntesis angulares (<etiqueta>).

2. En un fichero XML hay solo un elemento raíz.

3. Los elementos y los atributos son sensibles a las mayúsculas y minúsculas (<etiqueta> $\neq<$ ETIQUETA $>$ ).

4. Para realizar un fichero "bien formado" los elementos XML deben anidarse correctamente (<etiqueta1 > <etiqueta2 > </etiqueta2 > </etiqueta1 $>$ ).

5. Los valores de los atributos deben estar siempre entre comillas (<etiqueta atributo="valor" $>$ ).

6. Cada elemento XML debe tener una etiqueta de cierre (<etiqueta ></etiqueta>) $\mathrm{o}$, en referencia a las marcas "vacías", añadiendo una slash después del nombre de la etiqueta (<etiqueta/>).

\section{LA CODIFICACIÓN TEI}

Una vez realizadas estas aclaraciones, vamos a proceder a analizar la codificación TEl que ha sido adaptada al etiquetado del Cancionero: dado que TEl es un lenguaje basado sobre $\mathrm{XML}$, un documento TEI-XML empieza siempre con un prólogo XML: ningún elemento puede aparecer antes de esta declaración, como se puede ver en la tabla 1.

\section{TABLA 1}

$1:<$ ?xml version $=" 1.0$ " encoding $=$ "utf-8" ?>

2:

3: <? xml-stylesheet href=" cGM_transform.xsl" type="text/xsl" ?>

Sus funciones son las de declarar que es un fichero XML, la versión del lenguaje que se utiliza (la 1.0), y especificar qué caracteres de codificación se utilizan (aquí he elegido UTF-8, una combinación de caracteres UNICODE que utiliza de 8 a 32 bit para codificar cada carácter). La declaración de la línea 3 enlaza el documento a una hoja de estilo XSL (cGM_transform.xsl), creado para transformar este fichero en un documento HTML, como se verá más adelante.

Después del prólogo está el elemento raíz de cada documento TEl: entre la etiquetas $<$ TEl $>$ (el nodo raíz) y </TEl> se encuentra todo el contenido del documento, a partir del encabezado (tabla 2, líneas 6-22), donde están todos los metadatos sobre el texto codificado.

El <teiHeader> tiene un solo elemento obligatorio, llamado <fileDesc $>$, que es el elemento responsable de contener tanto la información sobre el fichero XML-TEl, como los detalles de la fuente primaria. 


\section{TABLA 2}

$1:<$ TEl $>$

2:

3: <?xml-model href="esquema_cancionero_manrique.xml"type="application/xml"

4: schematypens="http://relaxng.org/ns/structure/1.0"?>

5:

6: <teiHeader>

7:

8: <fileDesc >

9: <titleStmt >

10: <title>cancionero_gomez_manrique.xml</title>

11: $<$ /titleStmt $>$

$12:$

13: $<$ publicationStmt $>$

$14:<\mathrm{p}></ \mathrm{p}>$

15: $</$ publicationStmt $>$

16:

17: <sourceDesc $>$

18: $\langle\mathrm{p}></ \mathrm{p}>$

19: /sourceDesc $>$

20: </fileDesc $>$

21:

22: </teiHeader>

La marca <titleStmt> contiene la información sobre el título del documento, que está dentro de un elemento más específico llamado $<$ title $>$; $<$ publicationStmt $>$ recoge información sobre la publicación digital, que debe organizarse en párrafos $(<p>)$, mientras que la etiqueta < sourceDesc > contiene información sobre la fuente original.

Entre las líneas 3 y 4 la información que aparece sirve para enlazar el documento a un esquema especifico, que establece cuáles son los elementos y los atributos que se pueden utilizar, de forma que el documento resultante sea válido y conforme a un esquema previamente generado con la herramienta "Roma" (http://www.tei-c.org/ Roma/), una sencilla aplicación desarrollada para generar esquemas creada por el consorcio TEl. 
FIGURA 1

TEI Roma: generating customizations for the TEI

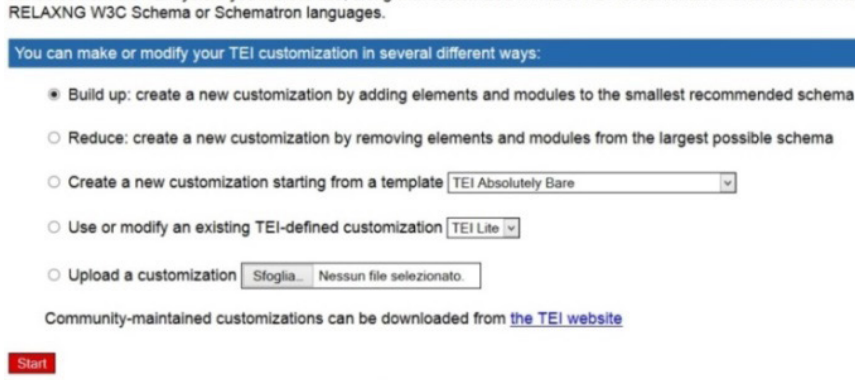
RELAXNG W3C Schema or Schematron languages.

You can make or modify your TEl customization in several different ways:

- Bulld up: create a new customization by adding elements and modules to the smallest recommended schema

Reduce: create a new customization by removing elements and modules from the largest possible schema

- Create a new customization starting from a template TEI Absolutely Bare

Use or modify an existing TEl-defined customization TEl Lite -

Upload a customization Stoglia. Nessun fle selezionato.

Community-maintained customizations can be downloaded from the TEl website

TEI Roma is a tool for working with TEI customizations. A TEl customization is a document from which you can generate a schema defining which elements and attributes from the TEI system you want to use, along with customized HTML or PDF documentation of it. The schema generated can be expressed in any of DTD.

FIGURA 2

TEI Roma: generating validators for the TEI

\section{Set your parameters}

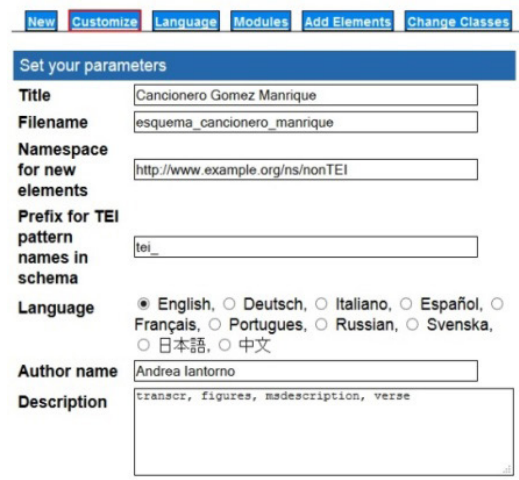

Save

La sección "description" de la figura 2 es importante, porque aquí se puede hacer una lista de los módulos de TEl que formarán el esquema: hemos insertado transcr (transcription), para la representación de fuentes primarias (manuscritos, por ejemplo); figures, que se utiliza para etiquetar ilustraciones y otras imágenes; msdescription 
(manuscript description), un módulo que sirve para proporcionar información detallada acerca de fuentes primarias escritas a mano $y$, obviamente, verse).

La segunda sección de un documento TEI-XML es la del texto, que empieza con el elemento <text> (tabla 3, línea 1), que puede contener además otras sub-secciones, como <front>, donde se marcan elementos paratextuales que preceden el texto), $<$ body $>$, que contiene el cuerpo del texto $y<$ back $>$, que se puede utilizar para añadir índices y apéndices.

\section{TABLA 3}

\section{1: $<$ text $>$}

2:

3: <body $>$

4:

5: <!-- página 11 -->

6: $<$ div xml:id="MP3-2">

7:

8: $\langle\lg n=" 1$ "type="octava" rhyme="abbacddc" $>$

9: $<\mathrm{n}=$ "1" $><$ hi rend="illuminated" $>\mathrm{P}</$ hi $>$ or quanto la $<$ rhyme label="a" $>$

10: oçiosidad $</$ rhyme $>,</ l>$

11: $<\mathrm{l} n=$ "2" $>$ amado muncho de $<$ rhyme label="b" $>$ my $</$ rhyme $>,</$ l $>$

$12:<\mathrm{n}=$ "3" $>$ es causa, según $<$ rhyme label $=$ "b" $>$ oy $</$ rhyme $>,</ l>$

13: $<\mathrm{l} n=$ "4" $>$ de pensar muncha $<$ rhyme label="a" $>$ maldad $</$ rhyme $></$ l $>$

$14:<$ n $=$ "5" $>$ e solo por $<$ rhyme label="c" $>$ esquiuar $</$ rhyme $></ l>$

15: $<$ n $=$ "6" $>$ aquesto, $<$ rhyme label="d" $>$ consideré $</$ rhyme $></ l>$

16: $<\mathrm{l} n=$ "7" $>$ esta quistión que no $<$ rhyme label $=$ "d" $>$ sé $</$ rhyme $></ l>$

17: $<\mathrm{l} n=$ "8" $>$ por trobas vos $<$ rhyme label $=$ "c" $>$ preguntar $</$ rhyme $>$. $</$ l $>$

18: $</ \lg >$

En el caso que nos ocupa, la información se contiene principalmente en la etiqueta $<$ body $>$.

Como puede verse, también aparecen en los comentarios en el propio etiquetado entre <!--y --> ) que contienen el número de la página del Cancionero codificada.

Además, se han registrado los números de codificación del índice de Dutton utilizando los elementos < div> (división de texto), que en TEl marca "una subdivisión del paratexto inicial, del cuerpo del texto o del paratexto final" (http://www.tei-c.org/release/doc/teip5-doc/es/html/ref-div.html), y los atributos @xml:id, para identificar unívocamente cada etiqueta y respetando siempre la denominación del Corpus de Dutton (línea 6).

Las líneas 8-18 contienen la codificación de la primera estrofa del cancionero: utilizando TEl cada grupo de versos, entonces también las estrofas, debe ser marcado 
entre las etiquetas <lg> (line group): en la marca inicial se pueden insertar atributos para añadir información sobre la estrofa codificada, como en la línea 8, donde se encuentra el número de la estrofa de la sección "MP3-2" ( $n=" 1$ "), así como la información del número de versos que la componen (type="octava") y su esquema métrico, utilizando el atributo @rhyme. Cada verso está marcado por una etiqueta $<\mathrm{l}>$ (line), numerada a través del atributo @n. Para enseñar el esquema métrico he marcado todas las palabras finales de los versos con el elemento <rhyme> y su atributo @label, "que identifica que parte del esquema rítmico representa la serie de caracteres en cuestión" (http://www.tei-c.org/ release/doc/tei-p5-doc/es/html/ref-rhyme.html) (línea 9).

En la codificación del primer verso, la primera letra (una P mayúscula) está marcada por la etiqueta $<$ hi $>$ (highlighted), que sirve para indicar "una palabra o frase gráficamente diferente del resto del texto que la circunda" (http://www.tei-c.org/release/doc/tei-p5-doc/ es/html/ref-hi.html) y en efecto, si se observa la primera página de una de las ediciones del Cancionero (figura 3), se puede ver como la primera letra está iluminada y por eso he utilizado el atributo @rend (rendering, para especificar los detalles de la gráfica) con el valor illuminated.

FIGURA 3

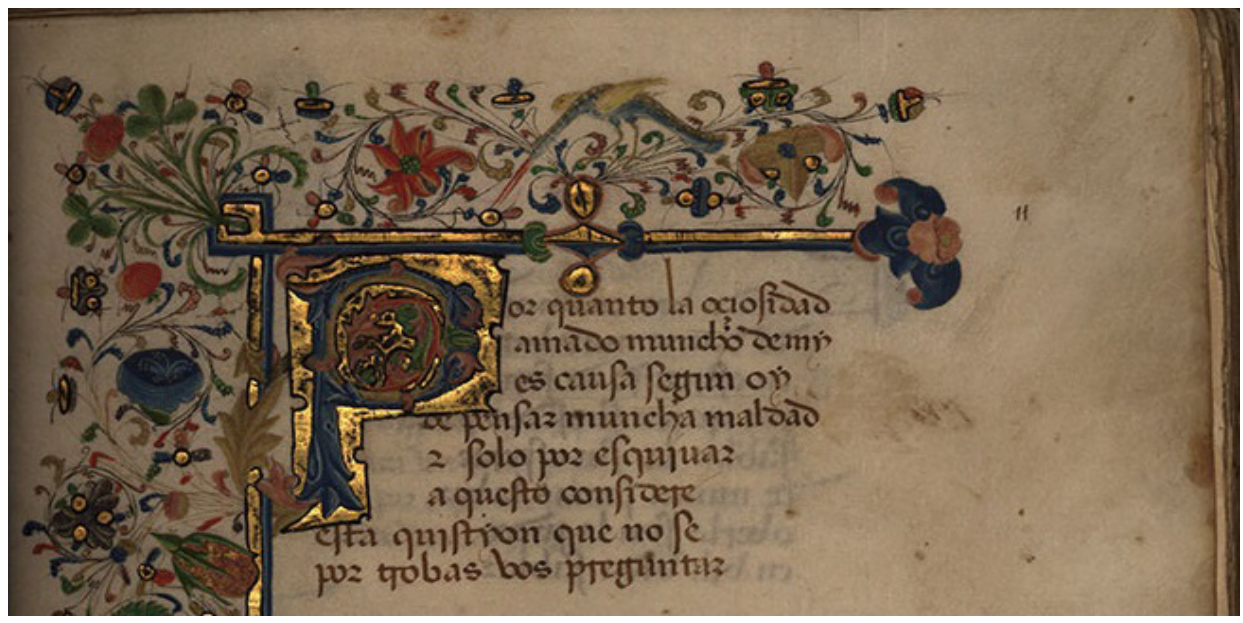

Para reportar cada salto de página TEl ofrece el elemento $<\mathrm{pb} />$ (page brake), como enseña la línea 6 de la tabla 4, donde está marcada la frontera entre las página 11 y 12.

\section{TABLA 4}

$1:<\mathrm{n}=$ "23" $>$ ¿quál es a vuestro $<$ rhyme label="d" $>$ entender $</$ rhyme $></$ l $>$

$2:<\mathrm{n}=$ "24" $>$ d'estas cosas la $<$ rhyme label="c" $>$ mejor $</$ rhyme $>:</ l>$

3: $</ \lg >$

4: 
5: <!-- página $12-->$

6: $<\mathrm{pb} \times \mathrm{xml}: \mathrm{id}=" 12 \mathrm{r} " />$

7:

8: $<\lg n=$ "4" type="octava" rhyme="abbacddc" >

9: $<\mathrm{ln}=$ "25" $>$ Syendo vos $<$ rhyme label="a" $>$ enamorado $</$ rhyme $></$ l $>$

10: $<\mathrm{ln}=$ "26" $>$ de dama muy $<$ rhyme label="b" $>$ virtuosa $</$ rhyme $>,</ l>$

$11:<\mathrm{n}=$ "27" $>$ en estremidad $<$ rhyme label="b" $>$ fermosa $</$ rhyme $>$, $<$ /l $>$

$12:<\mathrm{n}=$ "28" $>$ por quien fuésedes $<$ rhyme label="a" $>$ penado $</$ rhyme $>,</ l>$

En algunas canciones hay una estrofa final, precedidas por la palabra "Fyn" o "Ffyn" (figura 4, por ejemplo) y para etiquetar este fenómeno textual se ha insertado una marca $<$ note $>$ (tabla 5, línea 6).

\section{FIGURA 4}

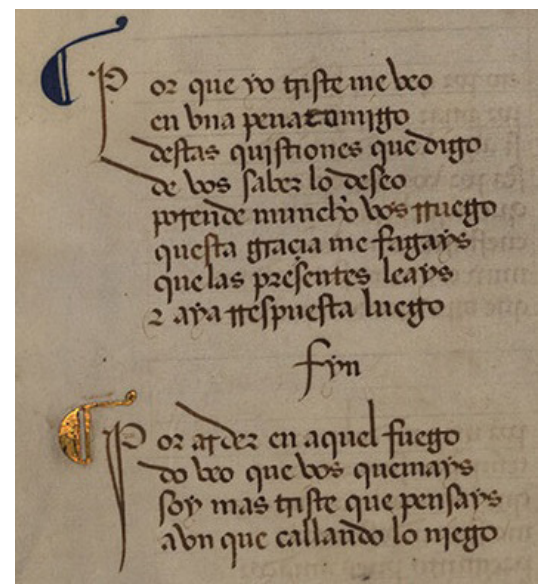

\section{TABLA 5}

$1:<\mathrm{n}=$ "38" $>$ qu'esta graçia me $<$ rhyme label="d" $>$ fagáys $</$ rhyme $>$. $</$ l $>$

$2:<\mathrm{n}=$ "39" $>$ que las presentes $<$ rhyme label="d" $>$ leáys $</$ rhyme $></ l>$

$3:<\mathrm{n}=" 40$ " $>$ e aya rrespuesta $<$ rhyme label $=$ "c" $>$ luego $</$ rhyme $>$. $</$ l $>$

4: $</ \lg >$

5:

6: $<$ note place $=$ "inline" $>$ Fyn $<$ /note $>$

7:

8: <lg n="6" type="cuarteta" rhyme="abba" >

9: $<\mathrm{l} n=$ "41" $>$ Por arder en aquel $<$ rhyme label="a" $>$ fuego $</$ rhyme $></$ l $>$

$10:<\mathrm{n}=$ "42" $>$ do veo que vos $<$ rhyme label="b" $>$ quemáys $</$ rhyme $>,</ l>$

$11:<\mathrm{l}=$ "43" $>$ soy más triste que $<$ rhyme label="b" $>$ pensáys $</$ rhyme $>,</$ l $>$ 
$12:<$ n="44" >avnque callando lo $<$ rhyme label="a" $>$ niego $</$ rhyme $>$. $<$ /l $>$

13: $</ \lg >$

14:

15: $<$ /div $>$

Se puede también especificar la posición de la nota, a través del atributo @place (en este caso con valor inline). En los facsímiles del cancionero se pueden encontrar algunas composiciones precedidas por el nombre del autor escrito en rojo, seguido por el título de la canción, en el margen izquierdo del papel o en una línea completa.

FIGURA 5

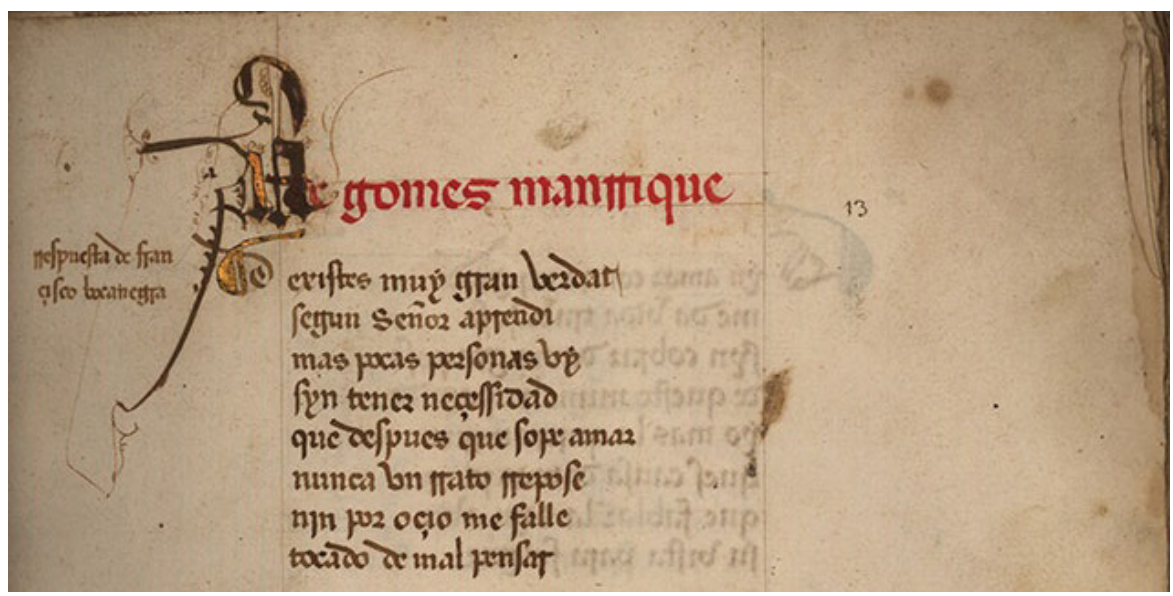

En el fichero XML se han codificado estos rasgos estilísticos con las etiquetas <opener> (tabla 6, línea 3) y <head> (tabla 6, línea 5): la primera "agrupa la fecha, autoría, fórmula introductiva o de saludo o expresiones similares utilizadas al inicio de una división textual [...]" (http://www.tei-c.org/release/doc/tei-p5-doc/es/html/ref-opener.html), mientras que $<$ head > puede contener"cualquier tipo de encabezamiento, por ejemplo el título de una sección" (http://www.tei-c.org/release/doc/tei-p5-doc/es/html/ref-head.html).

\section{TABLA 6}

1: $<$ div xml:id="MP3-3">

2:

3: <opener> De Gómez Manrique </opener>

4:

5: <head >

6: Respuesta de Fran- $<\mathrm{lb} />$

7: çisco Bocanegra

8: </head $><$ !-- Al margen izquierdo --> 
9:

10: $<\lg n=$ "1" type="octava" rhyme="abbacddc" >

$11:<\mathrm{n}=$ "1" $>$ Dexistes muy gran $<$ rhyme label $=$ "a" $>$ verdat $</$ rhyme $>,</$ l $>$

$12:<\mathrm{n}=$ "2" $>$ según, señor, $<$ rhyme label="b" $>$ aprendí $</$ rhyme $>,</ l>$

$13:<\mathrm{n}=$ "3" $>$ mas pocas personas $<$ rhyme label="b" $>$ vy $</$ rhyme $><$ l $>$

14: $<$ I n="4" $>$ syn tener $<$ rhyme label="a" $>$ neçessidad $</$ rhyme $>;</ l>$

15 : $\left\langle\mathrm{ln}={ }^{\prime \prime} 5^{\prime \prime}>\right.$ que después que sope $<$ rhyme label="c" $>$ amar $</$ rhyme $></$ l $>$

16: $<$ n $=$ "6" $>$ nunca vn rrato $<$ rhyme label="d" $>$ rreposé $</$ rhyme $></ l>$

17: $<\mathrm{l} n=$ "7" $>$ nin por oçio me $<$ rhyme label="d" $>$ fallé $</$ rhyme $></ l>$

18: $<\mathrm{l} n=$ "8" $>$ tocado de mal $<$ rhyme label="c" $>$ pensar $</$ rhyme $>$. $</ \mid>$

19: $</ \lg >$

En la línea 6 aparece el elemento <lb/> (line break), que "marca el comienzo de una nueva línea [...]" (http://www.tei-c.org/release/doc/tei-p5-doc/es/html/ref-lb.html): en la figura 5 en efecto se puede ver que la línea del título se interrumpe, rompiendo la palabra "Françisco" en "Fran" y "çisco". En algunas canciones en cambio, hay estrofas introducidas por anotaciones en el margen izquierdo.

\section{FIGURA 6}

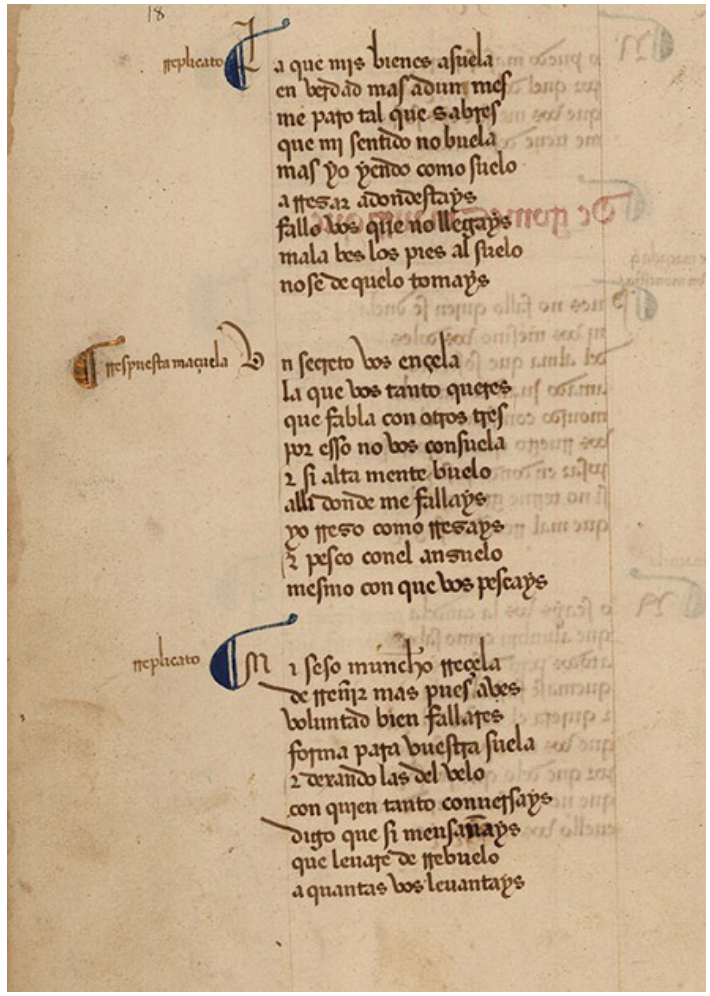


Estas anotaciones, a diferencia del título, están etiquetadas mediante <add> (addition): la función de <add > es la de contener "letras, palabras o frases introducidas en el texto por el autor, transcriptor, glosador o corrector" (http://www.tei-c.org/release/doc/ tei-p5-doc/es/html/ref-add.html).

También aquí se puede utilizar el atributo @place para especificar la posición del fenómeno textual codificado (tabla 7, línea 5).

\section{TABLA 7}

1: $<!--M P 3-7$ página 18-->

2: $<\mathrm{pb}$ xml:id="18r"/>

3:

4:

5: $<$ add place $="$ margin-left" $>$

6: Replicato

7: $<$ /add $>$

8: $<$ !- Al margen izquierdo -->

9:

10:

11: $<\lg n=" 3$ " type="novena" rhyme="abbacddcd" $>$

$12:<\mathrm{n}=" 19$ " $>$ La que mis bienes $<$ rhyme label="a" $>$ asuela $</$ rhyme $></ l>$

\section{LA HOJA DE STILO XSL}

La desventaja de los lenguajes de marcado semántico es la poco agradable presentación de datos al ojo humano, como se ha visto en las muestras de código XMLTEl: para resolver este problema se utilizan los lenguajes de presentación, que definen cómo presentar los datos al usuario.

Para mostrar los datos XML (con posibilidad de insertar imágenes, utilizar colores, modificar la disposición de contenidos etc...) hemos optado por transformar la codificación TEl en un fichero HTML y luego utilizar también una hoja de estilo CSS (Cascading Style Sheets, hojas de estilo en cascada) para trabajar sobre las marcas HTML: para lograr esto he utilizado XSLT (eXtensible Style Sheets Transformation, de la familia $\mathrm{XSL}$ ), un lenguaje para convertir documentos XML en otros ficheros, como texto simple, HTML, XHTML (eXtensible Hyper Text Markup Language) y también XML.

Las etiquetas de XSL son parecidas a las de HTML o XML, pero están siempre precedidas por xsl:, como se puede ver en la tabla 8.

\section{TABLA 8}

$1:<$ ?xml version $=" 1.0$ " encoding $="$ utf- 8 " ? >

2: 
3: <xsl:stylesheet version="1.0"

4: xmlns:xsl="http://www.w3.org/1999/XSL/Transform">

5:

6: $<$ xsl:output method="html" version="5" indent="yes"

7: xmlns:xsl="http://www.w3.org/1999/XSL/Transform"/>

Siendo un estándar relacionado con XML, se recomienda que la hoja de estilo XSL comience con una declaración XML (línea 1), aunque esto no es necesario, mientras que la marca <xsl:stylesheet $>$ de la línea 3 es obligatoria y su valor debe ser una versión válida de XSL (actualmente la única es la 1.0).

La etiqueta <xsl:output> de la línea 5 es muy importante, porque permite elegir el formato de salida del documento y debe aparecer como un nodo hijo del elemento <xsl:stylesheet> (como en este fichero) o de <xsl:transform>: gracias al atributo @ method he elegido crear un documento HTML, mientras que con @versión se selecciona su versión (la número 5).

Siguiendo la descripción del documento "cGM_transform.xsl" se encuentra el elemento <xsl:template> (tabla 9, línea 1), que indica el comienzo de la transformación porque pone en relación (por el atributo @match) la hoja de estilo con la raíz del fichero XML (/TEl, la barra indica que este es el nodo raíz): cada vez que se quiere manipular o llamar un elemento del fichero XML, se utiliza XPath (XML Path Language), un lenguaje de XSL (como XSLT) "used to navigate through elements and attributes in an XML document" (http://www.w3schools.com/xpath/).

\section{TABLA 9}

1: <xsl:template match $=" / T E I ">$

2:

3: <html>

4:

5:

6: <head >

7: $<$ meta http-equiv="content-type" content="text/html" charset="utf-8" />

8: <title>cGM_transform.xsl </title>

9: $</$ head $>$

10:

11:

$12:<$ body $>$ 
El elemento <xsl:template> contiene reglas que se aplican cuando se empareja un nodo especificado y su atributo @match, que "is used to associate the template with an XML element" (http://www.w3schools.com/xsl/el_template.asp).

La etiqueta $<$ html $>$ de la línea 3 indica el comienzo del documento HTML: entre las líneas 6 y 9 está el encabezado (<head $>$ ) del fichero, que contiene metainformaciones (contenidas en la marca $<$ meta $>$ ) sobre el contenido del fichero (http-equiv="contenttype" y content="text/html"), los caracteres de codificación (charset="utf-8") y el nombre del documento (línea 8).

Desde la línea 12 hay el cuerpo (<body>) del documento "cGM_transform.xsl", donde hay una única sección (<div>) que contiene algunas líneas de código XSL, como se puede leer en la tabla número 10.

\section{TABLA 10}

1: <body>

2:

$3:<\operatorname{div}>$

4:

5:

6: $<$ xsl:for-each select="/TEl/text/body/div" >

7:

8: <xsl:copy-of select="'!'/>

9:

10: </xsl:for-each $>$

11:

12:

13: </div $>$

14:

15:

16: </body $>$

17:

18: $</$ html $>$

19:

20: </xsl:template $>$

21:

22: </xsl:stylesheet $>$

En la línea 6 aparece la marca <xsl:for-each>, que se utiliza en XSLT para seleccionar todos los elementos XML de un conjunto especificado de nodos, y estos se seleccionan del fichero XML a través del atributo @select y se escribe como su valor el nombre del 
elemento que se quiere manipular, o la ruta XPath para navegar por el documento XML y recuperar el elemento deseado: "a location path can be absolute or relative. An absolute location path starts with a slash (/) and a relative location path does not. In both cases the location path consists of one or more steps, each separated by a slash" (http://www. w3schools.com/xpath/xpath_axes.asp).

En las líneas de código se puede ver como se utiliza un camino de localización "absoluta", porque todo el recorrido se desarrolla a partir del nodo raíz, para llegar finalmente a los elementos div que en el file XML contienen las diferentes canciones; para mostrarlas se copia todo el contenido de los nodos div utilizando <xsl:copy-of> y escribiendo un punto como valor de su atributo @select: esta marca XSL permite de hacer una copia en profundidad de los nodos seleccionados, porque "namespace nodes, child nodes, and attributes of the current node are automatically copied [...]" (http:// www.w3schools.com/xsl/el_copy-of.asp). El documento se cierra con el elemento </ xsl:stylesheet> de la línea 22.

La transformación final del documento XML en HTML se ha realizado de forma automática a través del software oXygen, como indica la marca <html > en la línea 1 de la figura 7. La figura 8 es el documento HTML resultado de esta transformación.

\section{FIGURA 7}

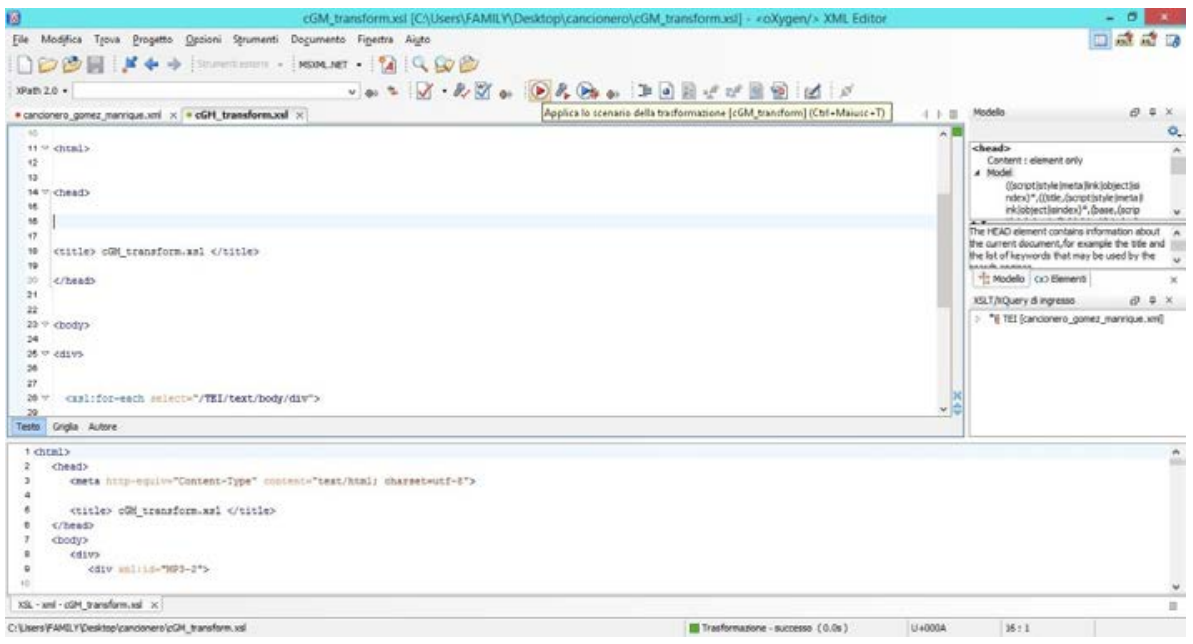




\section{FIGURA 8}

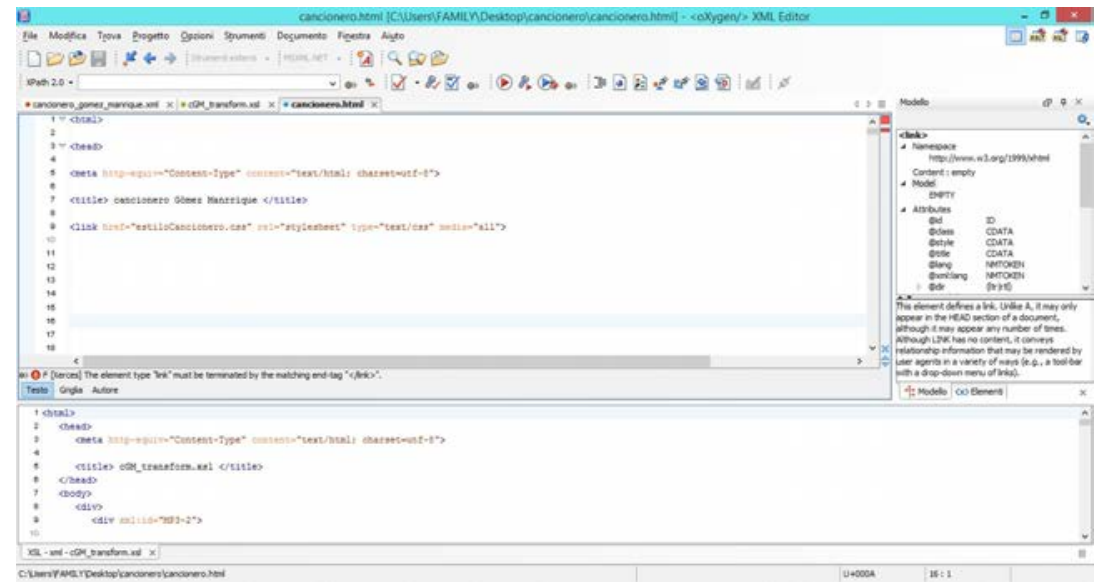

\section{CREAR UNA PÁGINA HTML5}

Sin embargo, para obtener un documento HTML 5 sin errores de validación ni de funcionamiento en los navegadores, se han tenido que realizar algunos cambios: he sustituido las etiquetas TEI por marcas HTML; luego en cada etiqueta he añadido el atributo @class, que sirve para asociar la misma propiedad CSS a varios elementos del mismo tipo, tomando como valor el nombre de la etiqueta TEl que codifica ese fenómeno textual, para no perder la referencia a la codificación semántica (tabla 3), como puede verse en la tabla11.

\section{TABLA 11}

1: $<$ !-- texto a la derecha -->

2: $<$ div id="canciones" $>$

3:

4: <!--MP3-2 p.11-->

5: $<$ div id="pag11" class="pag_bre" >

6:

7:

8: $<$ div class $=" \mid g^{\prime \prime}>$

9: $<$ p id="p11v1" class="l" $><$ span class="bef" $>1</$ span $>$

10: $<$ span class $=$ "hi" $>\mathrm{P}</$ span $>$ or quanto la

11: $<$ span class $=$ "a" $>$ o\&ccedil;iosidad $</$ span $>,</$ p $>$

12:

13: $<$ p id="p11v2" class $=" \mid "><$ span class="bef" $>2</$ span $>$ amado muncho de 
14: $<$ span class $=$ "b" $>$ my $</$ span $>,</ p>$

15:

16: $<$ p id="p11v3" class="l" ><span class="bef" $>3</$ span $>$ es causa, seg\&uacute;n 17: $<$ span class $="$ "b" $>$ oy $</$ span $>,</ p>$

18:

19: $<$ p id="p11v4" class="l" $><$ span class="bef" $>4</$ span $>$ de pensar muncha

20: $<$ span class $=$ "a" $>$ maldad $</$ span $></$ p $>$

21:

22: $<$ p id $=$ "p11v5" class $=" \mid "><$ span class $="$ bef" $>5</$ span $>$ e solo por

23: $<$ span class $=$ "c" $>$ esquiuar $</$ span $></$ p $>$

24:

25: $<$ p id="p11v6" class="l" $><$ span class $=$ "bef" $>6</$ span $>$ aquesto,

26: $<$ span class $=$ "d" $>$ consider\&eacute; $</$ span $></$ p $>$

27:

28: $<$ p id="p11v7" class $=" \mid "><$ span class="bef" $>7</$ span $>$ esta quisti\&oacute;n que 29: $\quad$ no $<$ span class $=" d ">$ s\&eacute; $</$ span $></$ p $>$

30:

31: $<$ p id="p11v8" class="l" $><$ span class="bef" $>8</$ span $>$ por trobas vos

32: $<$ span class $=$ "c" $>$ preguntar $</$ span $>.</$ p $>$

33: $</$ div $>$

La tabla 11 (primera estrofa de la primera canción) muestra la conversión de las marcas TEl lg en < div class="lg" > (línea 8), las I en $<$ p class="l" $>$ (línea 9), <hi $>$ en $<$ span class="hi" $>$ (línea 10) y, en la línea 11, < rhyme label="a" $>$ en $<$ span class="a" $>$ (span se utiliza para agrupar inline-elements en un documento HTML).

En esta transformación se han cambiado además otras etiquetas, como <note>, que se ha convertido en $\langle$ p class $=$ "note" $\rangle$, $\langle$ opener $\rangle$ se ha hecho $\langle$ p class="opener" $\rangle$, $<$ head $>$, que se ha covertido en $<$ p class="head" $>$ y $<$ add $>$ en $<$ p class="add" $>$.

A continuación se han insertado las imágenes de los facsímiles, etiquetadas utilizando $<$ figure $>$ y <img $>$, y que se han colocado a la izquierda de la página web, mientras que a la derecha se ha colocado el texto etiquetado por HTML. Todos los elementos se han formateado utilizando una hoja de estilo CSS ("estiloCancionero.css"). 


\section{FIGURA 9}

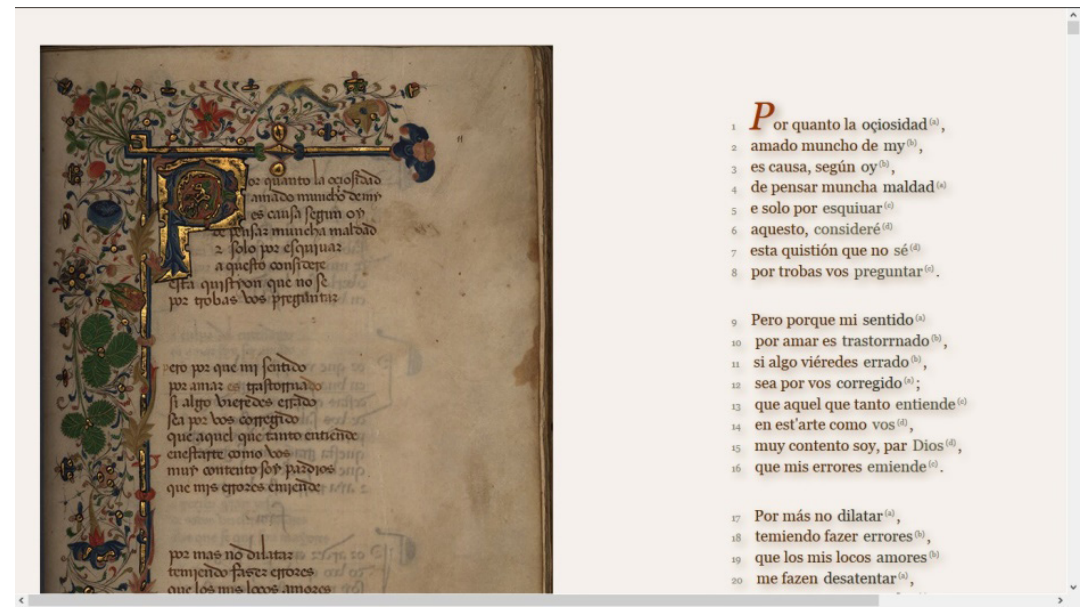

En este proyecto concreto, es especialmente importante el denominado parallel transcription method (método de transcripción en paralelo), puesto que resulta la principal aportación del mismo. Para ello, se han etiquetado gráficamente las imágenes, marcando su correspondencia en el texto mediante marcas <div> y asociándolas un identificador unívoco para posicionar los marcos de cada línea con una clase CSS llamada "marco" para dibujar los bordes (tabla 12 y 13 ).

\section{TABLA 12}

1: <!-- marcos de cada linea (:hover) - Parallel Transcription Method -->

2: $<$ div id="marcos" $>$

3:

4: $<$ div id="marcos_p11">

5: $<$ !- p.11 marcos -->

6: $<$ div id="p11_v1" class="marco" $></$ div $>$

7: $<$ div id="p11_v2" class="marco" $></$ div $>$

8: $<$ div id="p11_v3" class="marco" $></$ div $>$

9: $<$ div id="p11_v4" class="marco" $></$ div $>$

10: $<$ div id="p11_v5" class $=$ "marco" $></$ div $>$

11: $<$ div id="p11_v6" class="marco" $></$ div $>$

12: $<$ div id="p11_v7" class="marco" $></$ div $>$

13: $<$ div id="p11_v8" class="marco" $></$ div $>$

14: [...] 


\section{TABLA 13}

1:. marco

2: \{

3: border-radius:10px;

4: cursor:crosshair;

5: background-color:transparent;

6: border-color:\#FFFF66;

7: border-width:2px;

8: border-style:groove;

9:\}

10:

11: [...]

12:

13: \#p11_v1

14: \{

15: position:absolute;

16: top:192px;

17: width:630px;

18: height:30px;

19: left:40px;

20:\}

El código CSS se recoge en la tabla 13: todos los < div> de la clase "marco" ${ }^{6}$ tienen el mismo tipo de borde redondeado (border-radius:10px) y de color (código \#FFFF66) (línea 6), con estilo "groove" y con 2 píxeles de espesor, mientras que el identificador (\#) p11_v1 posiciona el marco del primero verso de manera absoluta, con una distancia de 192 píxeles del borde superior y 40 píxeles a la izquierda de la página web: "an absolute position element is positioned relative to the first parent element that has a position other than static. If no such element is found, the containing block is <html>" (http:// www.w3schools.com/css/css_positioning.asp).

El resultado de este trabajo de etiquetado puede verse en la figura siguiente.

6 Sabemos que "marco" es una clase por el punto que le precede en el código. 
FIGURA 10

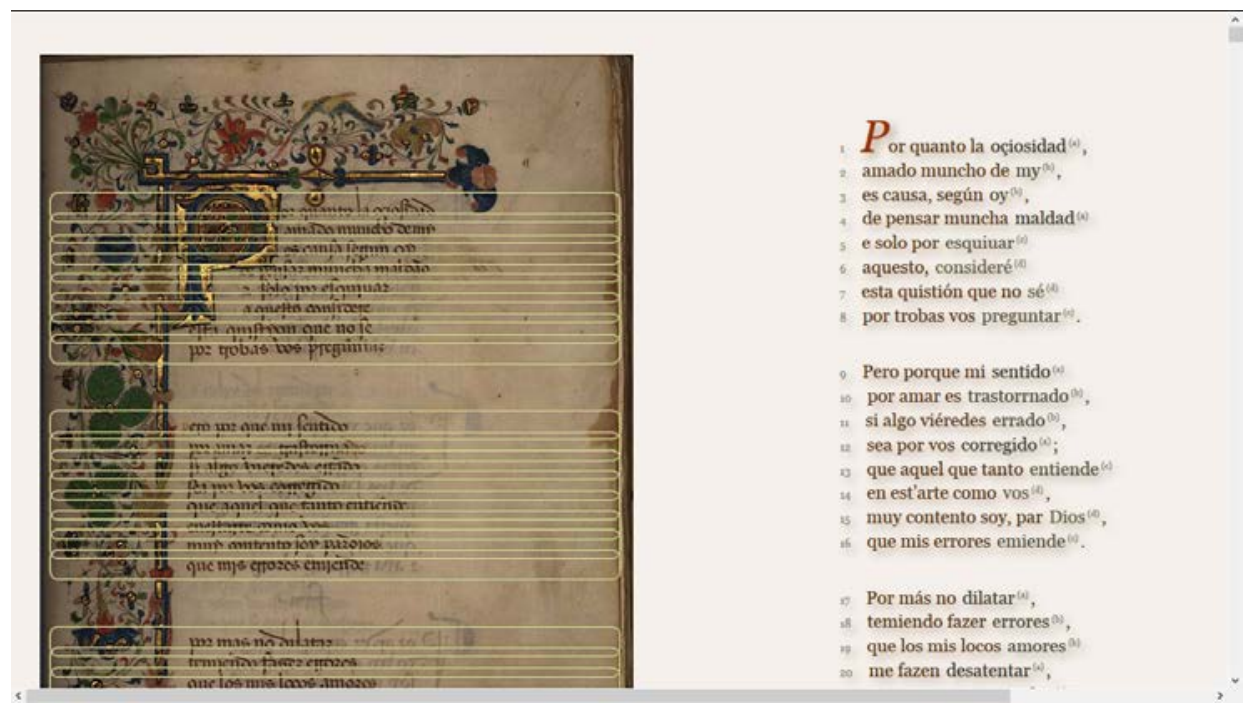

A esto se ha añadido un efecto de aparición sobre la palabra de este marco cuando se pasa el ratón por encima (figura 11). Esto se hace mediante :hover (tabla 14, línea 7) una pseudo-clase que se usa para definir un estado especial de un elemento, tal y como se describe: "the :hover selector is used to select elements when you mouse over them" (http://www.w3schools.com/cssref/sel_hover.asp)

\section{TABLA 14}

1: .marco

2: \{

3: border-radius:10px;

4: cursor:crosshair;

5: \}

6:

7: marco:hover

8: \{

9: background-color:transparent;

10: border-color:\#FFFF66;

11: border-width:2px;

12: border-style:ridge;

13:\} 


\section{FIGURA 11}

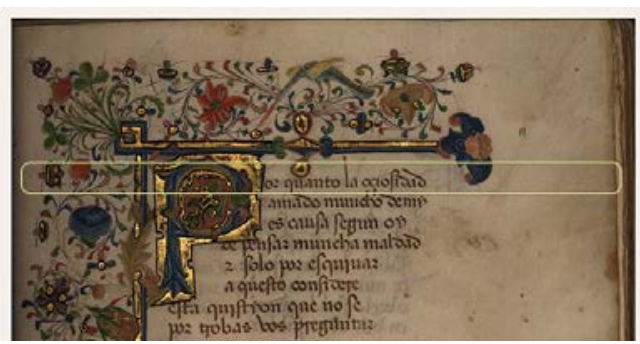

$P_{\text {or quanto la oçiosidad }}$,

amado muncho de my ${ }^{00}$

es causa, según oy ${ }^{(4)}$,

de pensar muncha maldad

e solo por esquiuar

aquesto, consideré (i)

esta quistión que no sét

por trobas vos preguntar ${ }^{\mathrm{s}}$

Esta misma clase :hover se ha utilizado además para resaltar a través del ratón los versos del texto a la derecha (figura 12).

\section{TABLA 15}

1: p:hover

2: \{

3: font-weight:bold;

4: font-style:italic;

5:\}

Así, cada vez que se pone el ratón sobre un elemento marcado por $<p>$ el texto aparecerá en negrita (font-weight:bold;) y en cursivo (font-style:italic;), como sucede en la primera línea de la imagen número 12.

\section{FIGURA 12}

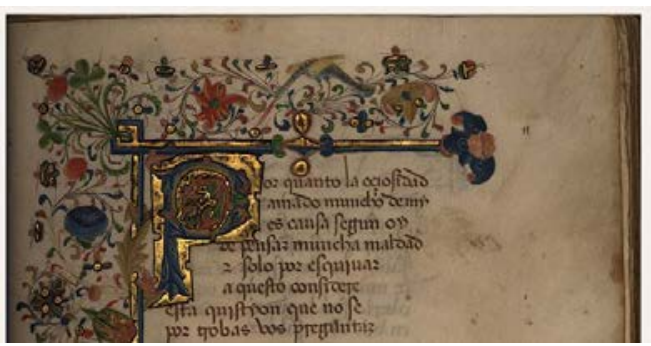

Por quanto la oçiosidad amado muncho de my ${ }^{\text {s }}$

es causa, según oy ${ }^{\text {아 }}$,

de pensar muncha maldad $*$

e solo por esquiuar

aquesto, considerécu

esta quistión que no sét

por trobas vos preguntar io

El siguiente paso ha sido la creación de un enlace entre las líneas de las imágenes y las del texto, para resaltar instantáneamente y en paralelo los versos que se corresponden. Para conseguir este objetivo se ha utilizado jQuery, que, al igual que JavaScript es un lenguaje creado para hacer páginas Web interactivas y dinámicas, pero más sencillo de usar ${ }^{7}$

7 Se puede leer más sobre jQuery en http://www.w3schools.com/jquery/jquery_intro.asp,. 
Para añadir jQuery en una página Web se puede utilizar una versión alojada en Google, Microsoft o jQuery.com o se puede descargar el archivo jQuery en versión de escritorio. En este caso se ha utilizado la CDN de Google, escribiendo en la sección <head> del fichero HTML el código que puede leerse en las líneas 9, 10 y 11 de la tabla 16.

\section{TABLA 16}

1: <!DOCTYPE html>

2: $<$ html $>$

3:

4: <head >

5: <meta http-equiv="Content-Type" content="text/html; charset=utf-8">

6: <title> Cancionero Gómez Manrique </title>

7: <link href="estiloCancionero.css" rel="stylesheet" type="text/css"

8: media="all" $>$

9: <script

10: src="http://ajax.googleapis.com/ajax/libs/jquery/1.11.2/jquery.min.js" $>$

11: </script>

12:

13: $<$ script src="funciones_PTM.js" $><$ /script $>$

14: $</$ head $>$

La etiquetas <script> (línea 9 y 13) marcan el inicio del código JavaScript, mientras que los cierres </script> marcan su final: como se puede ver, estas etiquetas tienen la apariencia de las etiquetas HTML; la línea 13 vincula el documento HTML al fichero externo "funciones_PTM.js", donde se encuentra todo el código jQuery.

Para crear el método de transcripción paralela, se han seleccionado los identificadores de cada verso del texto a la derecha, asegurando que el paso del ratón sobre ellos resalta en el mismo momento las líneas correspondientes a la izquierda, como puede verse en la tabla 17. Afortunadamente, una de las ventajas de jQuery es que "ofrece una técnica muy completa para seleccionar y trabajar con una colleción de elementos: los selectores CSS" (Mc Farland, David Sawyer, 2012: 152).

\section{TABLA 17}

1: /* Funciones jQuery creadas para implementar el PTM */

2: \$(document).ready(function()\{

3:

4: /*pagina $11 * /$

5: /*text-image*/

6: \$('\#p11v1').hover(function() 
7: \{

8: \$('\#p11_v1').addClass('res1')

9: \}

10: ,function()

11: \{

12: \$('\#p11_v1').removeClass('res1')

13: \});

14:

15: /*text-image*/

16: \$('\#p11_v1').hover(function()

$17:\{$

18: \$('\#p11v1').addClass('res2')

19: \}

20: , function()

21: \{

22: \$('\#p11v1').removeClass('res2')

23: \});

Las líneas 6-13 de la tabla 17 contienen la primera función: en la 6 he seleccionado el identificador del primero verso a la derecha, a través del carácter $\$$, y he utilizado el jQuery hover() Method para especificar que la función se aplicará solo si se pone el ratón sobre el verso indentificado por \#p11v1: cuando esto sucede, instantáneamente se aplica, gracias a addClass(), al verso identificado por \#p11_v1 (lo del verso de la imagen a la izquierda) la clase CSS ".res1", que tiene las mismas propiedades de la pseudo-class .marco:hover de la tabla 14, mientras que si se quita el ratón (,function()) del verso a la derecha se retira (removeClass()) también la clase ".res1" en lo de la imagen.

El mismo procedimiento se repite para todos los versos de las imágenes de la izquierda (líneas 16-23). El resultado se puede ver en la figura siguiente.

FIGURA 13

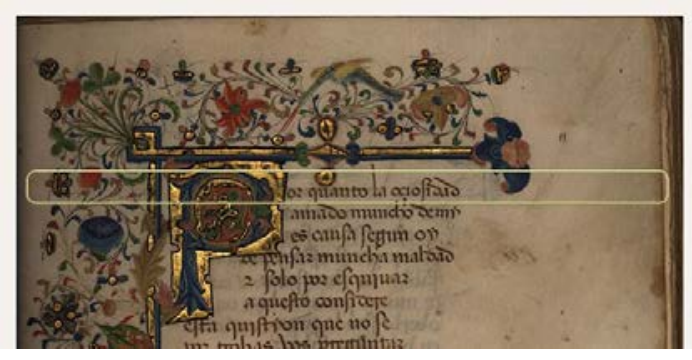

P amado muncho de my(i), es causa, según oy ${ }^{(0)}$,

de pensar muncha maldad ${ }^{(\infty)}$

e solo por esquiuar

aquesto, consideré (t) $^{(0)}$

esta quistión que no sé(i)

por trobas vos preguntar ${ }^{(e)}$. 


\section{REFERENCIAS BIBLIOGRÁFICAS}

CSS :hover Selector. http://www.w3schools.com/cssref/sel_hover.asp [18/07/2015].

CSS LAYOUT - The position property. http://www.w3schools.com/css/css_positioning.asp [18/07/2015].

IANTORNO, Andrea. Cancionero digital de Gómez Manrique, http://www.realbiblioteca.es/ gomez_manrique/cancionero.html [18/08/2015]. Código del proyecto disponible en https://github.com/Andrealantorno/Cancionero-digital-de-G.-Manrique.

McFARLAND \& SAWYER, D. (2012). JavaScript y jQuery. Madrid: O'Reilly.

OXIGEN XML Editor. http://www.oxygenxml.com/[18/07/2015].

TEl <add>. http://www.tei-c.org/release/doc/tei-p5-doc/es/html/ref-add.htm/[18/07/2015].

TEl < div>. http://www.tei-c.org/release/doc/tei-p5-doc/es/html/ref-div.html [18/07/2015].

TEl <hi>. http://www.tei-c.org/release/doc/tei-p5-doc/es/html/ref-hi.html [18/07/2015].

TEl <lb>. http://www.tei-c.org/release/doc/tei-p5-doc/es/html/ref-lb.html [18/07/2015].

TEI <opener>. http://www.tei-c.org/release/doc/tei-p5-doc/es/html/ref-opener.html

[18/07/2015].

TEl <head>.http://www.tei-c.org/release/doc/tei-p5-doc/es/html/ref-head.html [18/07/2015].

TEl <rhyme>. http://www.tei-c.org/release/doc/tei-p5-doc/es/html/ref-rhyme.html

[18/07/2015].

TEl Roma. http://www.tei-c.org/Roma/[18/07/2015].

TEl verse. $h$ ttp://www.tei-c.org/release/doc/tei-p5-doc/es/html/VE.html [18/07/2015].

The Digital Vercelli Book. http://vbd.humnet.unipi.it/beta/\#105r [18/07/2015].

XPath Axes. http://www.w3schools.com/xpath/xpath_axes.asp [18/07/2015].

Xpath Tutorial. http://www.w3schools.com/xpath/[18/07/2015].

XSLT <xsl:copy-of>Element. http://www.w3schools.com/xsl/el_copy-of.asp [18/07/2015].

XSLT <xsl:template> Element. http://www.w3schools.com/xsl/el_template.asp

[18/07/2015].

Recibido el 17 de octubre de 2015.

Aceptado el 30 de octubre de 2015. 